\title{
Traduire
}

Ine autre perspective sur $r$ tadadciction

Revue française de la traduction

245 | 2021

Regards sur l'interprétation

\section{Le corps en interprétation. Dimension non verbale des discours interprétés}

\section{Monika Chwalczuk et Jean-Michel Benayoun}

\section{(2) OpenEdition}

1 Journals

Édition électronique

URL : https://journals.openedition.org/traduire/2409

DOI : 10.4000/traduire.2409

ISSN : 2272-9992

Éditeur

Société française des traducteurs

Édition imprimée

Date de publication : 5 décembre 2021

Pagination : 11-26

ISSN : 0395-773X

\section{Référence électronique}

Monika Chwalczuk et Jean-Michel Benayoun, « Le corps en interprétation. Dimension non verbale des discours interprétés », Traduire [En ligne], 245 | 2021, mis en ligne le 05 décembre 2021, consulté le 23 décembre 2021. URL : http://journals.openedition.org/traduire/2409; DOI : https://doi.org/10.4000/ traduire.2409 


\section{Le corps en interprétation}

Dimension non verbale des discours interprétés

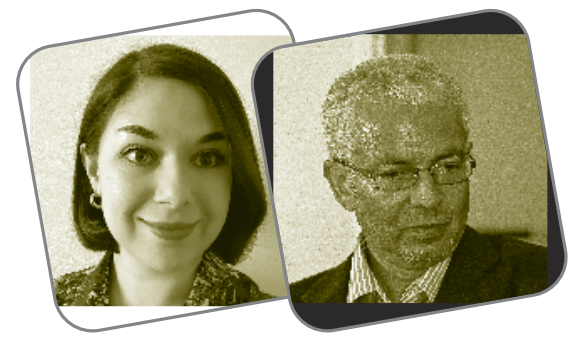

Monika Chwalczuk
Jean-Michel Benayoun

À la différence de ce qui se passe en interprétation de conférence, l'interprétation en service public redistribue, par la simple présence physique des intervenants, les relations entre textes-source/textes-cible, entre messages émis/ messages recus, entre chacun des niveaux de la communication eux-mêmes. Ici, l'interprète est présent: on le voit, on l'entend et l'on guette les signaux non verbaux qu'il produit ou reproduit. C'est par l'intermédiaire de son corps, de son visage et de leurs expressions que s'établissent, de manière consciente ou inconsciente, les premiers degrés de la compréhension.

Les gestes, le regard et les mimiques faciales jouent un rôle réel dans la transmission du sens, en fournissant des informations qui, capitalisées, deviennent complémentaires du message verbal. À partir d'un corpus multimodal, combinant les données recueillies au cours d'entretiens (menés auprès de 12 interprètes professionnels), d'enquêtes (échantillon de 60 répondants), mais aussi de séquences vidéo enregistrées (corpus de 13000 secondes annotées), nous nous proposons d'analyser comment les expressions non verbales 
participent de l'intelligence cognitive à l'œuvre au sein des événements interprétés.

Les recherches récentes menées par les gestualistes européens et internationaux (McNeill, 2005; Müller, 2018; Ferré, 2019; Azaoui et Tellier, 2020) intègrent les objets d'étude de traductologues spécialistes de l'interprétation (Navarro et Benayoun, 2016; Angelelli, 2019; Pöchhacker, 2020). Aujourd'hui, les psychologues cognitivistes (Aussems et Kita, 2017; Goldin-Meadow, 2017) utilisent des corpus multimodaux pour réfléchir, entre autres, aux stratégies de compensation du phénomène bien connu de saturation cognitive (Gile, 2009 ; Pointurier, 2016).

Dans le cadre de cet article, nous proposerons, dans un premier temps, une synthèse des principales théories de la communication multimodale et nous envisagerons des points de convergence théorique pour proposer un modèle fonctionnel à même de rendre compte du rôle de la voix et du corps dans les interactions interprétées. Ensuite, après avoir élaboré une typologie des productions non verbales, des gestes et des phénomènes multimodaux, nous analyserons son efficacité dans la stratégie de compensation de la surcharge cognitive.

En combinant les avancées de domaines tels que la sociolinguistique, la traductologie, la linguistique de corpus, la psychologie cognitive et les études gestuelles, nous nous posons pour objectif de montrer comment la perspective multimodale non seulement contribue à une meilleure compréhension des opérations mentales soutenant le processus d'interprétation, mais participe également de l'efficacité des dispositifs multilingues.

\section{Les enjeux de la présence physique de l'interprète en service public}

À l'époque où toute communication humaine est soumise à une omniprésente dématérialisation, nous risquons de perdre de vue ce que cela signifie d'être présent par son corps. Celui-ci, loin d'être un simple siège des attributs les plus nobles d'un être humain, à savoir l'esprit, la pensée ou les sentiments, constitue incontestablement une partie matérielle de notre existence et, donc, une partie importante des éléments que nous utilisons pour communiquer. Si nous 
nous référons à ce qu'écrit l'école de Palo Alto (Watzlawick et al., 1972), nous postulons que l'émission d'informations par le corps humain n'est pas un simple processus que nous pouvons activer ou désactiver sur demande, mais que tout comportement, toute action peut être source d'informations.

Couvrant une mosaïque de lieux d'intervention (écoles ou centres de protection maternelle et infantile, tribunaux, structures administratives destinées aux migrants, commissariats de police, services hospitaliers), l'interprétation en service public, comme peu de métiers de traduction aujourd'hui, exige de l'interprète qu'il soit physiquement présent lors de la séance, ce qui lui donne accès à tous les éléments du contexte qui participent à la construction du sens. Ainsi, lors des missions d'interprétation sur site, entrent en jeu tous les éléments constitutifs de l'éthos (Benayoun et Navarro, 2014) des interactants: les signes visibles d'âge, le genre, la taille ou la couleur de peau, mais aussi les parures ou les attributs liés à des métiers précis, comme un stéthoscope ou une blouse. Si, en interaction en face-à-face, les éléments factuels statiques font appel aux représentations mentales idiosyncratiques des interlocuteurs, le changement de perspective, du sujet parlant au récepteur du message, implique un nouveau filtrage des informations reçues, faisant ainsi basculer les données statiques analysées inconsciemment en un ensemble d'interprétations dynamiques, reliant alors le processus communicationnel aux fondements de la cognition.

Tout uniforme constitue un outil d'identification permettant de dissocier l'institution représentée par le fonctionnaire de l'être humain qui l'incarne. Si l'interprète n'arbore aucun signe distinctif confirmant son statut professionnel (Benayoun et Navarro, 2014; Pointurier, 2016), les usagers prêtent attention à tout signe d'ordre personnel. Percevoir l'interprète comme une personne, et non pas comme un agent anonyme d'une institution, permet d'établir une relation de confiance et d'empathie, même si cela constitue un risque certain.

La spécificité du travail de l'interprète en service public consiste à se déplacer d'un lieu d'intervention à un autre, tel un «loup solitaire» ${ }^{1}$, couvrant souvent plusieurs missions

1. Métaphore employée par l'un des interprètes travaillant chez ISM Interprétariat lors d'un entretien. 
de différents types en une seule journée. Or, cette absence d'association claire au lieu d'intervention, combinée au manque d'éléments visibles de tenue professionnelle, risquent de gêner la reconnaissance d'une profession qui reste aujourd'hui encore peu visible dans l'espace public. Nous constatons de plus que l'interprète travaillant sur site, dépourvu d'anonymat, est tenu de supporter personnellement tout le poids d'un échange en présentiel: interactions à grande charge émotionnelle, ou interprétation pour des usagers violents et dangereux.

Les perquisitions d'habitude prennent place à $6 \mathrm{~h}$ du matin. Je suis le premier derrière la porte devant un bonhomme de $150 \mathrm{~kg}$ avec des tatouages. Je ne suis pas briefé, je n'ai pas de gilet pare-balles, contrairement aux policiers. (...) J'ai juste le gilet pare-balles linguistique. II faut que je lui explique la situation avant qu'il me blesse. ${ }^{2}$

Ce témoignage, récolté lors des entretiens individuels, met en lumière des craintes pour la sécurité de l'interprète, renforcées par l'impossibilité de se cacher derrière un uniforme ou d'être protégé par les murs de son lieu de travail.

Une fois, je suis sorti de la salle d'audience et j'ai croisé le frère de la requérante pour laquelle je venais d'interpréter. II m'a dit. j'espère que t'as bien traduit, parce que sinon... et il a frappé son poing contre la paume de l'autre main. (...) C'est beaucoup plus simple de travailler par vidéoconférence que face à face, c'est plus simple de garder sa neutralité et de se protéger des situations comme ça. ${ }^{3}$

La pression, augmentée par la proximité physique, pousse certains interprètes à refuser des missions en présentiel au profit du travail à distance, limitant de facto tout contact avec les usagers en dehors des séances.

2. Interprète en arabe et anglais; entretien du 13/03/2020.

3. Interprète en espagnol, créole guadeloupéen, allemand; entretien du 10/05/2018. 


\section{Le rôle du non-verbal dans la co-construction du sens}

Depuis la théorie interprétative de la traduction (Seleskovitch et Lederer, 2001), nous savons que toute opération traduisante repose non sur la répétition du message-source dans une autre langue, mais sur la transmission du sens initial. Or, et pour aller au-delà, il nous appartient de nous demander comment ce sens se construit lors des échanges oraux. Si la présence physique de l'interprète ouvre accès au contenu verbal et à la texture de la voix, les signaux non verbaux dynamiques (gestes, postures, mimiques faciales, profondeur du regard) contribuent tous à la construction du sens du message-source. Comme le résument les gestualistes, "les productions langagières sont inséparables de l'imagerie mentale incarnée par les gestes qui accompagnent la parole de manière universelle et automatique ${ }^{4}$ (McNeill, $2005: 16$ ). Cette observation est à l'origine du concept d'énoncé multimodal (Kendon, 2009: 44) qui met en valeur le caractère complémentaire et indissociable des différents signaux activés lors de l'expression orale:

le sens de ce qu'un orateur souhaite dire se présente à lui tout entier, et la difficulté que l'orateur rencontre, s'il do it l'exprimer à travers la langue parlée, est d'organiser ce matériel d'une façon qui permet de le présenter de la manière linéaire qu'exige la parole. ${ }^{5}$

L'usage de multiples articulateurs - la bouche, les mains ou la tête - permet ainsi d'échapper aux contraintes de la linéarité de la parole prononcée. L'externalisation de la pensée sous forme de parole multimodale, ou bien le passage des concepts proto-langagiers existant en "langage de la pensée» (Fodor, 1975) à l'expression orale, exige un certain nombre d'opérations cognitives. Afin d'illustrer et de structurer les opérations mentales soutenant ce processus, les gestualistes mettent en avant différents modèles de communication multimodale (Ferré, 2019). Le modèle formulé

4. Nous traduisons.

5. Nous traduisons. 
par Krauss (Lexical Facilitation Model, Krauss et al., 2001) s'appuie sur celui de Levelt (1989), illustrant la production de la parole, et cherche à montrer comment le concept préverbal est rendu à la fois à travers la parole et le geste, chacun représentant différents traits sémantiques du même référent. Parallèlement, le modèle de de Ruiter (Sketch Model, de Ruiter, 2000), reposant également sur les travaux de Levelt ainsi que sur les modèles existants en théorie de l'information, propose que le geste et la parole proviennent tous deux de la même intention communicationnelle qui est prise en charge par le système cognitif de traitement des informations préverbales, lequel "décide » si la parole ou le geste est plus efficace pour représenter certains éléments sémantiques. Selon de Ruiter, la difficulté de retirer un mot de l'ensemble du lexique peut être reconnue par le système cognitif qui transmet dès lors plus de contenu sémantique vers la modalité visuelle-gestuelle; c'est pourquoi nous produisons plus de gestes quand nous avons du mal à formuler le message verbalement. Le modèle élaboré par Kita et Özyürek (Interface Model, 2003) avance que les gestes sont générés avant la formulation linguistique des concepts adjacents, ce qui expliquerait pourquoi la phase de préparation d'un geste précède souvent la prononciation du mot-clé correspondant. Enfin, le modèle radial de la production de l'énoncé multimodal présenté par Ferré (2019) organise les différents mécanismes de planification sur un plan atemporel, soulignant que leur mise en place serait difficile à structurer chronologiquement.

Tant que les modèles existants se focalisent sur les processus mentaux sous-jacents, ou bien sur la phase de production de la parole multimodale, les éléments activés lors de la réception de la parole sont absents des représentations schématiques. Afin de comprendre comment la présence et la prise en compte des éléments non verbaux peuvent influencer la réception du message-source, il faut partir du principe que la compréhension n'est pas un processus passif, mais qui requiert plutôt que le récepteur filtre les informations obtenues, contribuant ainsi à la construction du sens. En effet, un énoncé multimodal est une unité discursive, correspondant à l'ensemble des interactions au niveau tant verbal que non verbal, soumise à des processus dialogiques de 
négociation de sens qui impliquent les acteurs du discours et les contextes de sa production (Chwalczuk, 2021). Pour saisir le sens d'un tel énoncé, il est donc nécessaire de prendre en compte:1) le geste manuel, impliquant la position du membre employé et le mouvement adjacent; 2) combiné à la production non verbale signifiante simultanée (un regard significatif, une expression faciale ou une posture particulière). Ces variables doivent être analysées au regard de la situation de communication d'une part, des participants et de leurs visées communicationnelles respectives d'autre part. Une telle conception pragmatique du sens permet de concevoir les unités sémantiques multimodales non comme des signes, des valeurs constantes en interactions, mais comme des praxèmes (Siblot, 1997) dont la structure se rapproche plutôt d'une équation à résoudre en fonction des variables de l'interaction.

\section{Typologie des productions non verbales signifiantes et distribution dans le corpus}

Afin de vérifier quelles productions non verbales sont en réalité présentes dans les échanges interprétés en service public, nous avons construit le premier corpus multimodal relatif à ce domaine après une collecte des données descriptives provenant d'un questionnaire et d'entretiens avec les professionnels du métier. Sur plus de 12 heures d'enregistrement brut, nous avons sélectionné un ensemble de 13015 secondes, illustrant les interactions de 27 interprètes travaillant en 16 langues provenant de 8 familles différentes ${ }^{6}$. Compte tenu des difficultés considérables pour obtenir des données écologiques, nous avons combiné des enregistrements réalisés sur le terrain avec des interactions semi-contrôlées, filmées dans le cadre de la formation des interprètes et disponibles en libre accès. Ainsi construit, le corpus de 126 séquences vidéo présente cinq contextes distincts, notamment les séances de pédopsychiatrie et de formation civique (corpus écologique), ainsi que des échanges

6. Les familles de langues germaniques, finno-ougriennes, indo-iraniennes, malaïques, romanes, sémitiques, sinitiques et slaves. 
dans le milieu médical, social et policier (corpus libre accès). Nous avons annoté l'ensemble du matériel audiovisuel dans le logiciel ELAN en nous appuyant sur la typologie des productions non verbales présentée ci-après.

Après une analyse croisée des classements gestuels avancés par McNeill (2005), Cosnier (1982) et Poyatos (1997), nous avons confronté leur répertoire avec des extraits pilotes du corpus multimodal, ce qui a donné naissance à une nouvelle typologie adaptée aux échanges bidirectionnels interprétés en service public. Nous avons établi quatre catégories prépondérantes donnant lieu à 12 types gestuels annotés. Nous distinguons ainsi les gestes symboliques, fonctionnant sur la base d'une convention sociale, qui regroupent les étiquettes, tels les hochements et les secouements de tête, les haussements d'épaules, les serrements de mains et les autres emblèmes manuels. La deuxième catégorie porte sur les gestes illustrateurs, qui fournissent une représentation visuelle des éléments évoqués dans le discours ou bien qui servent à faciliter la production verbale par le recours aux gestes; elle comprend les gestes iconiques, les gestes iconiques abstraits et les gestes conceptuels. La troisième catégorie implique les gestes régulateurs d'échange ayant pour fonction la coordination des tours de parole et la sollicitation de l'attention de l'interlocuteur, la mise en valeur ou la désignation d'un élément évoqué verbalement. Ces fonctions peuvent être réalisées respectivement par les gestes phatiques, les battements et les gestes déictiques. Enfin, les comportements tels que les mimiques faciales, les jeux de regards, le toucher, les postures, les attitudes et le positionnement dans l'espace ne sont pas des gestes dans le cadre de la définition adoptée dans ce travail. Ils constituent néanmoins des éléments importants qui peuvent influencer considérablement certains échanges, c'est pourquoi nous décidons de les inclure sous la forme d'une quatrième et dernière catégorie regroupant les autres productions non verbales signifiantes non couvertes par les catégories précédentes. Il faut préciser que les sous-types de gestes iconiques abstraits et de serrement de mains ont été ajoutés à ce système au cours du processus d'annotation, ce qui était nécessaire pour rendre compte de certains phénomènes observables dans le corpus. 
Productions non verbales annotées dans le corpus multimodal

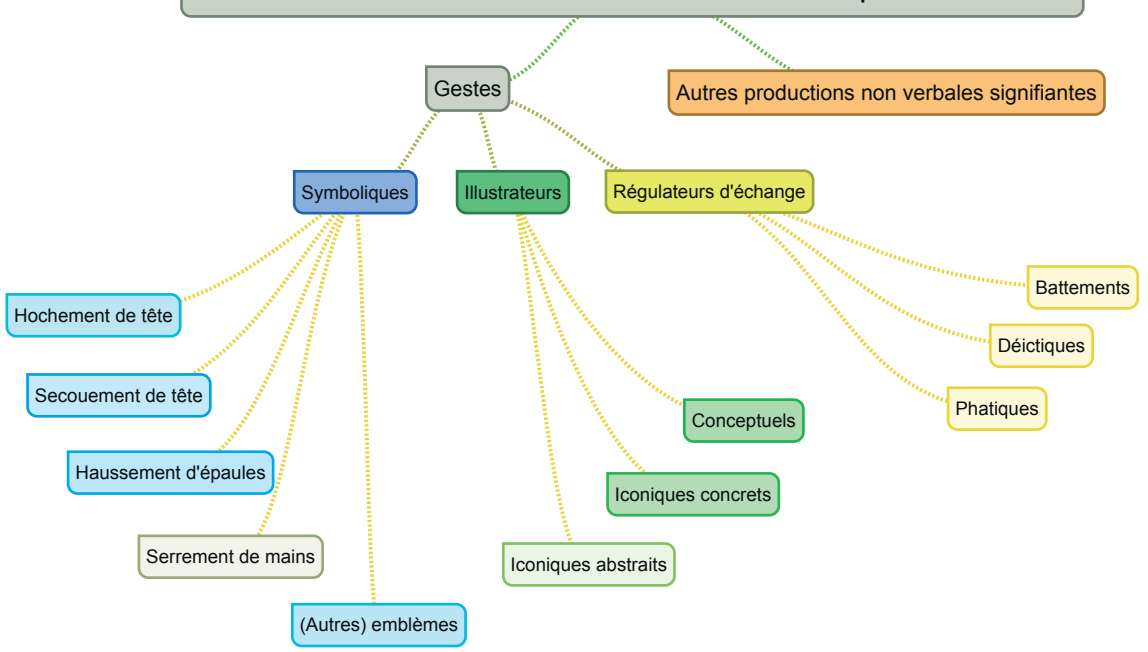

Typologie des gestes et autres productions non verbales en interprétation en service public.

Le corpus compte au total 12880 segments annotés, dont 5504 , soit $42,7 \%$, portent sur les gestes ou autres productions non verbales signifiantes, ce qui permet de constater sans équivoque que ces éléments jovent un rôle important. Cette observation s'avère fondamentale dans la mesure où les études en interprétation ont longtemps placé ce paramètre en marge du cadre analysé. Quant à la distribution des types gestuels parmi tous les participants des interactions analysées, contrairement à nos prévisions, elle se montre relativement stable au sein des cinq lieux d'interaction étudiés. Dans presque tous les contextes, le paysage gestuel est dominé par les mêmes types gestuels, notamment les gestes conceptuels $(22,3 \%)$, les hochements de tête $(21,4 \%)$ et les gestes déictiques $(15,1 \%)$, qui représentent au total près de $60 \%$ de tous les gestes du corpus.

En ce qui concerne la production gestuelle des 27 interprètes, le ratio geste/parole dans leurs contributions s'élève en moyenne à $46,84 \%$, ce qui signifie que les professionnels accompagnent de gestes presque la moitié de leurs 
interventions ${ }^{7}$. Les profils gestuels des interprètes travaillant dans les différents contextes présentent de fortes affinités. Ainsi, dans presque tous les contextes analysés, le trio des types dominants comprend toujours les gestes conceptuels (30\%), les gestes déictiques (18\%) et les hochements de tête (17\%). La répartition des gestes mis en place par les interprètes est donc compatible avec les paysages gestuels présentés par les échanges en général, ce qui démontre que l'usage des productions non verbales chez l'interprète est conditionné par les sujets abordés, par le contenu sémantique du message-source et par les gestes des participants.

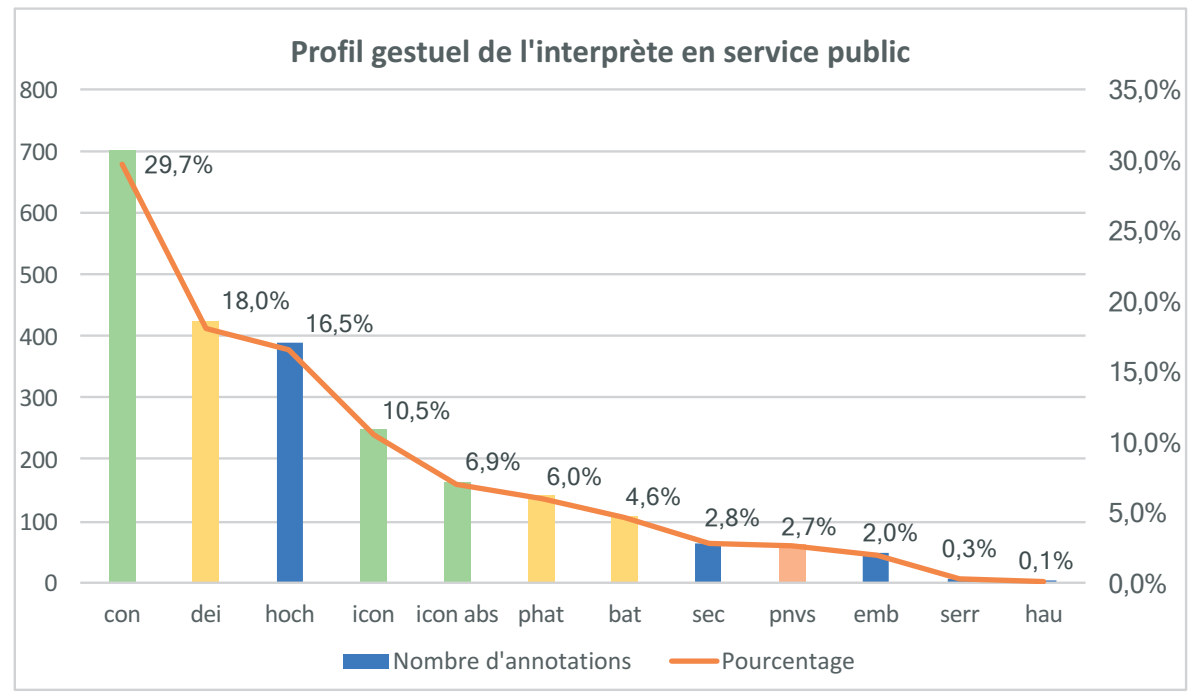

Fréquence d'usage des différents types gestuels par les interprètes.

Même si chacun de ces types relève, dans notre classement, d'une catégorie différente, il s'agit dans les trois cas de gestes qui mobilisent peu d'activité cognitive et ne relèvent pas entièrement de la volonté des sujets parlants. Autrement dit, l'usage assez libre et fréquent de ces types gestuels suggère que les interprètes n'ont pas conscience

7. Ce paramètre présente pourtant une forte variabilité idiosyncratique, allant de $9,29 \%$ chez un interprète sinophone en formation civique à 94,64\% chez un interprète arabophone assurant un suivi en pédopsychiatrie. Les données ne révèlent pourtant pas de corrélation entre le lieu d'interaction et la quantité de gestes produits par les interprètes. 
de s'en servir et les sollicitent plutôt de manière involontaire et automatique, ce qui est confirmé par les commentaires fournis dans le questionnaire et les entretiens. D'autres arguments justifiant la production fréquente des types gestuels dominants sont liés à leur relation au message verbal et à leurs fonctions communicationnelles. Les gestes conceptuels servent avant tout la production de parole, en la facilitant. Les hochements de tête, quant à eux, participent au feedback non verbal, indispensable dans les cultures occidentales pour assurer un développement fluide des interactions en face-à-face. En ce qui concerne les gestes déictiques, leur usage fréquent peut se justifier par deux arguments principaux. Premièrement, du point de vue cognitif, ils mettent en place la loi de désignation du référent présent, formulée par Cosnier (1997), permettant de connecter la pensée du gesticulateur au monde extérieur en vertu de la contiguïté, à travers le pointage des objets ou des personnes dans l'espace commun. De plus, ils transmettent un contenu non redondant par rapport à la parole, facilitant ainsi la désambiguïsation et la désignation précise et sans équivoque des référents mentionnés.

\section{La reproduction des gestes}

L'analyse qualitative du corpus permet d'observer non seulement que les interprètes sollicitent fréquemment leurs mains pour accompagner leur production verbale, mais aussi que les gestes présents dans leurs contributions constituent souvent la reproduction de gestes-clés réalisés par les orateurs. En effet, au sein des 126 séquences annotées, nous retrouvons 102 occurrences de reproduction de gestes originaux par les interprètes (Chwalczuk, 2021). Ces résultats prouvent qu'il ne s'agit pas d'exemples isolés, mais plutôt d'un phénomène récurrent en interprétation. Les types gestuels les plus fréquemment reproduits impliquent avant tout des gestes iconiques et des gestes déictiques; donc des productions qui apportent d'importantes informations non verbales pour compléter ou illustrer la parole. 


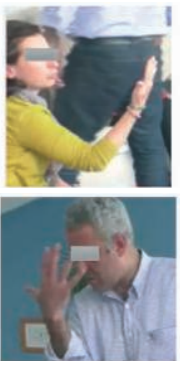

Pédopsychiatrie (français-arabe)

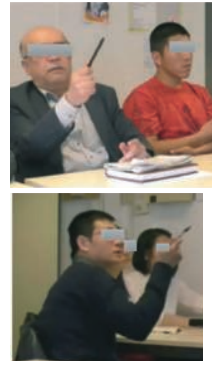

Formation civique (français-dari/mandarin

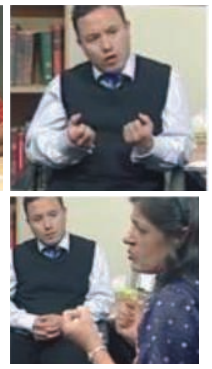

Corpus médical (anglais-tamil)
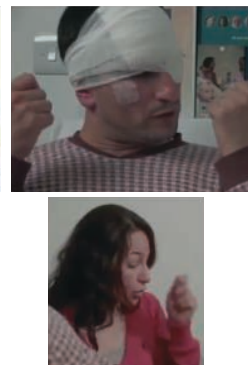

Corpus policier (anglais-arabe)

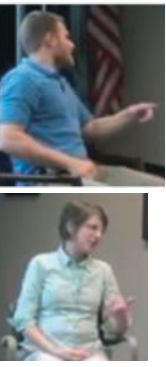

Corpus social (anglais-espagnol)

Reproduction des gestes iconiques et déictiques par les interprètes

Cette observation porte en elle des conclusions majeures quant à la dynamique interactionnelle des échanges interprétés en service public. 1) Compte tenu du caractère multimodal des énoncés-sources, leur reproduction à l'aide de plusieurs articulateurs semble plus naturelle et moins exigeante sur le plan cognitif que leur transmission à l'aide de mots seuls. 2) Les schémas de reproduction gestuelle suggèrent que l'interprète participe au processus de co-construction du sens multimodal qui, loin d'être passivement reçu, est plutôt élaboré de manière participative par les interlocuteurs (Di Paolo et De Jaegher, 2015; Azaovi et Tellier, 2020).

\section{Conclusion: le rôle des gestes dans la diminution de la charge cognitive}

Les arguments justifiant l'usage des gestes en communication interprétée trouvent leur place au sein même de la théorie du sens (Seleskovitch et Lederer, 2001), selon laquelle on distingue trois étapes universelles du processus d'interprétation: la compréhension du message-source, la déverbalisation des éléments sémantiques compris et la reproduction du discours en langue-cible.

Lors de la première phase, les gestes qui accompagnent le discours en langue-source facilitent la saisie des informations décrites puisqu'ils permettent de comprendre et de mieux mémoriser des mots utilisés. Ensuite, grâce à l'usage des stimuli visuels, tels que les gestes iconiques, l'interprète est en mesure de déverbaliser les informations plus facilement grâce au lien qui s'établit avec l'imagerie 
mentale (McNeill, 2005) et ses ressources cognitives, mobilisant ainsi d'autres dimensions que celle du langage: les sentiments vécus ou l'expérience personnelle, par exemple. En effet, en vertu des recherches sur la cognition par expérience corporelle (embodied cognition) (Di Paolo et De Jaegher, 2015), nous savons que le cerveau n'est pas le seul siège de la cognition et que les processus mentaux ont besoin d'être incarnés et mis en œuvre par le corps. Comme l'observe Bouvet (1997: 61), «toute appropriation du sens se réalise à travers l'expérience corporelle». Ainsi, puisque les mêmes éléments sémantiques sont transmis simultanément par les canaux verbaux et visuels, l'illustration des énoncés par les gestes met en place une sorte de double codage (Paivo, 1969), en stimulant de facto le processus de mémorisation (Aussems et Kita, 2017).

Enfin, la dernière phase d'interprétation consiste à se rappeler et à reformuler verbalement un contenu sémantique mémorisé plus tôt, ce qui se rapproche d'une tâche classique observée lors de protocoles expérimentaux par les chercheurs en études gestuelles. Selon eux, l'usage des gestes lors de la reproduction d'un récit est un processus naturel, qui favorise le rappel des détails (Cook et al., 2008), ce qui justifie l'abondance des gestes conceptuels dans notre corpus. De surcroit, les recherches empiriques en psycholinguistique cognitive (Gillespie et al., 2014) prouvent que la mise en place des gestes accompagnant la production orale permet de décharger la mémoire opérationnelle.

L'interprétation, métier encore très verbo-centrique, affiche traditionnellement une certaine méfiance face à l'usage des gestes, dont l'abondance est perçue comme peu professionnelle, mettant en danger l'impartialité de l'interprète par son potentiel expressif (Chwalczuk, 2021). II est primordial d'observer que, dans ce contexte, ce sont justement les gestes conceptuels qui occupent la place principale dans l'expression des professionnels. Vagues de forme, caractérisés par un mouvement continu des mains et un effort musculaire minimal, ils servent principalement à soutenir le flux de la parole, en facilitant «la mise en mots». Dépourvus de valeur communicationnelle stricto sensu, leur abondance dans la production des interprètes témoigne donc de leur potentiel à diminuer la charge cognitive liée à la production non 
seulement de la parole spontanée, mais aussi de la parole interprétée. Les travaux de Gile (2009) sur les modèles d'efforts en interprétation nous enseignent que l'interprète travaille souvent aux marges de ses limites de capacité de traitement, ce qui est décrit sous le nom de l'hypothèse de la corde raide. C'est pourquoi il est essentiel de prendre en considération toute technique permettant d'optimiser, voire de réduire, la consommation des ressources cognitives.

En conclusion, les résultats de cette recherche démontrent que l'approche multimodale permet d'étudier en profondeur tant les processus cognitifs soutenant les opérations traduisantes que les mécanismes de construction participative du sens mis en place au sein des interactions interprétées. À ce titre, il paraît nécessaire d'intégrer la perspective multimodale lors de la formation des futurs interprètes en service public, ce qui pourrait contribuer à l'amélioration du travail des dispositifs d'interprétation à l'avenir.

\section{monikachwalczuk@gmail.com jmb@eila.univ-paris-diderot.fr}

Docteure en Sciences de la Traduction, rattachée au laboratoire CLILLAC-ARP à I'Université de Paris, Monika Chwalczuk a soutenu sa thèse sous la direction du Professeur Jean-Michel Benayoun en mars 2021. Passionnée par la communication multimodale, elle mène depuis 2013 des travaux portant sur le rôle du non-verbal en interprétation dans les services publics. Interdisciplinaire, sa recherche se situe à la croisée de la traductologie, de la sociolinguistique, de la multimodalité, des études gestuelles et de la linguistique du corpus. Sa méthodologie comprend l'analyse des premiers corpus multimodaux des interactions interprétées en milieu social, médical et policier. 
Professeur de linguistique anglaise et de sociolinguistique, et membre du CLILLAC-ARP de I'Université de Paris, Jean-Michel Benayoun s'est spécialisé au cours des deux dernières décennies dans l'analyse des phénomènes traductologiques à l'œuvre au cours de l'interprétation en service public. Il est co-responsable du sous-axe Politiques linguistiques et interculturalité qui élabore des modèles théoriques d'analyse et de compréhension des sociétés multilingues et interculturelles. Chez Michel Houdiard Éditeur, il a publié 14 ouvrages dans la collection "Langues, Cultures, Représentations». En 2019, il crée la collection "InterCulturel» aux Éditions des archives contemporaines.

\section{SOURCES CITÉES}

ANGELELLI Claudia, Healthcare Interpreting Explained, Oxon/New York, Routledge, 2019 .

AUSSEMS Suzanne et KITA Sotaro, "Seeing Iconic Gestures While Encoding Events Facilitates Children's Memory of These Events ", in Child Development, 90(4), 2017, p. 1123-1137.

AZAOUI Brahim et TELLIER Marion, «Comment le corps coconstruit les discours et le sens ", in Travaux interdisciplinaires sur la parole et le langage, 6(1), 2020.

BENAYOUN Jean-Michel et NAVARRO Elisabeth, Interprétation et médiation. Deux objets pour un concept pluriel, Paris, Michel Houdiard Éditeur, 2014.

BOUVET Danielle, Le corps et la métaphore dans les langues gestuelles. À la recherche des modes de production des signes, Paris, L'Hartmann, 1997.

CHWALCZUK Monika, La gestualité co-verbale en interprétation dans les services publics. Analyse contextualisée d'un corpus multimodal, thèse de doctorat, Paris, Université de Paris, 2021.

COOK Susan et al., "Gesture Makes Learning Last», in Cognition, 106, 2008, p. 1047-1058.

COSNIER Jacques et al., Les voies du langage. Communications verbales, gestuelles et animales, Paris, Dunod, 1982.

DE RUITER Jan Peter, «The Production of Gesture and Speech», in McNEILL David (dir.), Language and Gesture, Cambridge, Cambridge University Press, 2000, p. 284-311.

DI PAOLO Ezequiel et DE JAEGHER Hanne, «Toward an Embodied Science of Intersubjectivity: Widening the Scope of Social Understanding Research », in Frontiers in Psychology, 6, 2015, p. 234-250.

FERRE Gaëlle, Analyse de discours multimodale. Gestualité et prosodie en discours, Grenoble, Univesrité Grenoble Alpes Éditions, 2019.

FODOR Jerry, The Language of Thought, Cambridge (Mass.), Harvard University Press, 1975. 
GILE Daniel, Basic Concepts and Models for Interpreter and Translator Training, Amsterdam/Philadelphia, John Benjamins, 2009.

GILLESPIE Maureen et al., «Verbal Working Memory Predicts Co-speech Gesture: Evidence from Individual Differences », in Cognition, 132(2), 2014, p. 174-180.

GOLDIN-MEADOW Susan et BRENTARI, Diane, «Gesture, Sign, and Language: The Coming of Age of Sign Language and Gesture Studies », in Behavioral and Brain Sciences, 40, 2017, p. 1-17.

KENDON Adam, «Kinesic Components of Multimodal Utterances », in Annual Meeting of the Berkeley Linguistics Society, 35(2), 2009, p. 36-53.

KITA Sotaro, «How Representational Gestures Help Speaking», in McNEILL David (dir.), Language and Gesture, Cambridge, Cambridge University Press, 2000, p. 162-185.

KITA Sotaro et ÖZYÜREK Asli, «What Does Cross-linguistic Variation in Semantic Coordination of Speech and Gesture Reveal? Evidence for an Interface Representation of Spatial Thinking and Speaking», in Journal of Memory and Language, 48(1), 2003, p. 16-32.

KRAUSS Robert et al., «Lexical Gestures and Lexical Access: A Process Model», in McNEILL David (dir.), Language and Gesture, New York, Cambridge University Press, 2001, p. 261-283.

McNEILL David, Gesture and Thought, Chicago, University of Chicago Press, 2005.

MÜLLER Cornelia, «Gesture and Sign: Cataclysmic Break or Dynamic Relations? », in Frontiers in Psychology, 9, 2018, p. 1-20.

NAVARRO Elisabeth \& BENAYOUN Jean-Michel, «Interprétation-médiation: une étude pragmatique de la désignation d'un nouveau métier», in PHILLIPSBATOMA Patricia \& XIANGYUN ZHANG Florence, Translation As Innovation. Bridging the Sciences and the Humanities, Victoria, TX, Dalkey Archive Press, 2016, p. 169-186.

PAIVO Allan, «Mental Imagery in Associative Learning and Memory», in Psychological Review, 76(3), 1969, p. 241-263.

PÖCHHACKER Franz, "Going Video: Mediality and Multimodality in Interpreting ", in SALAETS Heidi et BRÔNE Geert (dir.), Linking Up with Video: Perspectives on Interpreting Practice and Research, Amsterdam/Philadelphia, John Benjamins Publishing Company, 2020, p. 13-46.

POINTURIER Sophie, Théories et pratiques de l'interprétation de service public, Paris, Presses Sorbonne Nouvelle, 2016.

POYATOS Fernando, Nonverbal Communication and Translation: New Perspectives and Challenges in Literature, Interpretation and the Media, Amsterdam/Philadelphia, John Benjamins, 1997.

SELESKOVITCH Danica et LEDERER Marianne, Interpréter pour traduire, Paris, Didier Erudition, 2001.

SIBLOT Paul, «Nomination et production de sens: le praxème», in Langages, 127, 1997, p. 38-55.

WATZLAWICK Paul et al., Une logique de la communication, Paris, Éditions du Seuil, 1972. 